\title{
Mars Orbiter Laser Altimeter pulse width measurements and footprint-scale roughness
}

\author{
G. A. Neumann, ${ }^{1,2}$ J. B. Abshire, ${ }^{2}$ O. Aharonson, ${ }^{3}$ J. B. Garvin, ${ }^{4}$ X. Sun, ${ }^{2}$ and M. T. Zuber ${ }^{1,2}$ \\ Received 3 February 2003; revised 12 March 2003; accepted 25 March 2003; published 5 June 2003.
}

[1] The Mars Orbiter Laser Altimeter (MOLA) measured the pulse width and energy of altimetric laser returns during the course of two Mars years of operations. As secondary science objectives, MOLA obtains the footprint-scale roughness and the bidirectional reflectivity of Mars. MOLA underwent extensive preflight calibration and pulse measurements were monitored continuously in flight, but anomalous values of roughness have been inferred. A calibration of pulse widths using inflight data yields a slopecorrected roughness over $\sim 75$-m-diameter footprints that may be used for quantitative geomorphic surface characterization, required, for example, for landing site selection. The recalibration uses a total least-squares estimation of pulse characteristics that generalizes the method of Abshire et al. [2000]. This method, utilizing the timing at voltage threshold crossings and the area between crossings, accounts for observation errors and shows that surface roughness as small as $1 \mathrm{~m}$ can be resolved. INDEX TERMS: 5470 Planetary Sciences: Surface materials and properties; 5464 Planetary Sciences: Remote sensing; 5494 Planetary Sciences: Instruments and techniques. Citation: Neumann, G. A., J. B. Abshire, O. Aharonson, J. B. Garvin, X. Sun, and M. T. Zuber, Mars Orbiter Laser Altimeter pulse width measurements and footprint-scale roughness, Geophys. Res. Lett., 30(11), 1561, doi:10.1029/2003GL017048, 2003.

\section{Introduction}

[2] The Mars Orbiter Laser Altimeter (MOLA) [Zuber et al., 1992] became the first planetary ranging lidar on Sept. 15, 1997. In the succeeding 45 months, more than 600 million ranges were obtained, improving our knowledge of the topography of Mars by 3 orders of magnitude [Smith et al., 2001]. Altimetric shots provide information characterizing the target surface within the laser footprint, since surface height variations $h$ broaden the laser pulse by $2 h / c$ or $6.67 \mathrm{~ns}$ $\mathrm{m}^{-1}$, where $c$ is the speed of light. Garvin et al. [1999] and Smith et al. [2001] reported roughness estimates for early MOLA profiles and initial results during mapping, using pulse width and energy inversions based on analytic theory and preflight calibrations. However, the pulse width measurements of an internal diode standard were lower than preflight, and the observed ground pulse widths were often inconsistent

\footnotetext{
${ }^{1}$ Department of Earth, Atmospheric and Planetary Sciences, Massachusetts Institute of Technology, Cambridge, Massachusetts, USA.

${ }^{2}$ Laboratory for Terrestrial Physics, NASA/Goddard Space Flight Center, Greenbelt, Maryland, USA.

${ }^{3}$ Department of Geological and Planetary Sciences, California Institute of Technology, Pasadena, California, USA.

${ }^{4}$ NASA Headquarters, Washington, D. C., USA.
}

with theoretical system response. Moreover, inversions were sensitive to measurement errors. In this paper we present an inflight calibration of the pulse width and energy, and discuss the resulting implications for surface characteristics.

[3] To detect the wide range of pulse widths and energies coming from level surfaces, slopes as high as $67^{\circ}$, and clouds, MOLA's preamplified pulse entered four parallel filter channels. Only the first channel to trigger was recorded, the width of which usually corresponded to the best match to the returned pulse width. MOLA-1, lost on the Mars Observer Mission in 1993, had only the filter channels to distinguish differing surface characteristics. The instrument flown on the Mars Global Surveyor added a coarse measurement of the width of the pulse at the triggering voltage threshold, and of the pulse area, integrated from leading to trailing edge. Abshire et al. [2000] give a semianalytical solution for the received pulse energy and rootmean-square (RMS) pulse width from these measurements, assuming a Gaussian shape for the received pulse.

[4] MOLA's success came at a price. Reliability and cost constrained its design to be simple. Power and telemetry bandwidth restrictions precluded digitization of the returning laser waveforms, as later performed on the Shuttle Laser Altimeter built with MOLA spares [Garvin et al., 1998]. Sensitivity of the system was designed for reliable operation under worst-case signal conditions, with limited prior knowledge of what these might be, forcing the signal preamplifier to have high gain. Amplified pulses sometimes saturated the electronics and artificially prolonged the resulting waveform. A variable gain amplifier, as later flown on SLA-02, was not selected for reasons of risk avoidance.

[5] Even without preamplifier saturation, returns often exceeded their digital ranges in width and amplitude. Digital saturation of one or both measurements precludes a reliable estimate of pulse width and energy. Pulse width was primarily needed to correct the MOLA range to the centroid time of the returning pulse. Despite a threshold adjustment early in the mapping mission and a gradual decline in laser output, most pulses remained digitally saturated except over dark terrain or through clouds and dust.

[6] By the end of mapping, 200 million unsaturated ground returns at nadir incidence were obtained, covering the majority of the planet. We now discuss their pulse width measurement, their recalibration, an inversion method, and their interpretation in terms of footprint-scale surface roughness over sloping terrain.

\section{MOLA Pulse Measurement}

[7] Figure 1 shows the detection scheme on a single channel. The first channel to exceed its threshold setting $y$ triggers the range measurement. To compensate for background noise variability from night to day, flight software 


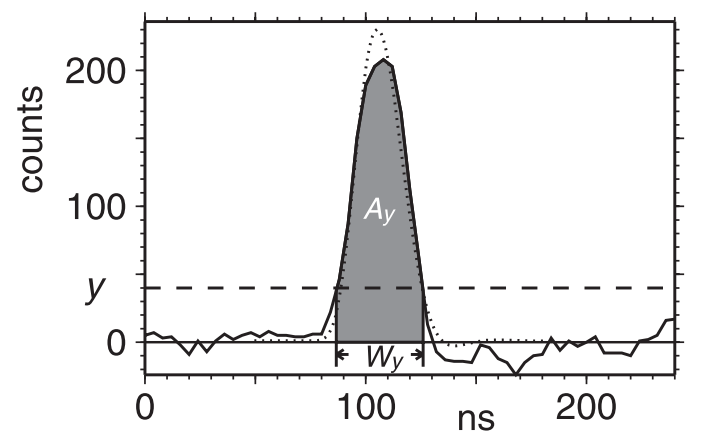

Figure 1. Pulse waveform from SLA-02. Darkened area represents energy $A_{y}$ measured between leading and trailing edges of pulse at threshold $y$ (dashes). System impulse response at this energy (dotted curve) constrains the minimum width of pulse $W_{y}$.

adjusts the thresholds on each channel once per second at settings from $0-255 \mathrm{mV}$, typically from $40-100 \mathrm{mV}$. The threshold on channel 1 was commanded to $245 \mathrm{mV}$ after 3 months of operation to mitigate pulse width saturation. After clocking the leading edge of the return, the electronics measure the duration of the pulse above threshold and the energy between threshold crossings using charge-time converters (QT100's), whose output is digitized to 6 and 8 bits precision, respectively. Each QT100 gives a number of counts linearly proportional (with an offset) to input. The width of the pulse between threshold crossings $W_{y}$ is given by

$$
W_{y}=a_{w}(i)\left(P_{w}-b_{w}(i)\right),
$$

where $P_{w}$ is the raw pulse width measurement, $a_{w}$ is the scale factor per least significant bit (LSB), $b_{w}$ is offset, and $i$ is the trigger channel. Table 1 gives original and recalibrated pulse width conversion factors for counts to time. Separate coefficients are required for the nonlinear response to short pulses. The energy measurement is linear with counts and, being a time integrated voltage, is less affected by system response bandwidth and noise than the pulse width.

\section{Inflight Pulse Width Calibration}

[8] Pulse width and energy of a diode test source remained fairly steady during mapping but pulse width was $3-7$ counts lower than preflight. Diode output varied with temperature, limiting measurement precision. A more precise calibration is

Table 1. System Response, Pulse Width Conversion Factors and Count Offsets by Filter Channel: Original and Recalibrated Values

\begin{tabular}{|c|c|c|c|c|c|}
\hline Channel & $\begin{array}{c}\text { integration } \\
\text { time (ns) }\end{array}$ & $\begin{array}{c}a_{w} \\
\text { (ns/count) }\end{array}$ & $\begin{array}{c}b_{w} \\
\text { counts }\end{array}$ & $\begin{array}{c}a_{w} \\
\text { (this study) }\end{array}$ & $\begin{array}{c}b_{w} \\
\text { (this study) }\end{array}$ \\
\hline \multirow[t]{3}{*}{1} & 20 & 3.6 & 7.4 & 3.6 & 4.45 \\
\hline & & $0.768^{\mathrm{a}}$ & -10.5 & $3.2^{\mathrm{b}}$ & 1.75 \\
\hline & & & & $2.0^{\mathrm{c}}$ & -3.2 \\
\hline \multirow[t]{2}{*}{2} & 60 & 7.79 & 5.3 & 8.0 & 3.5 \\
\hline & & & & $4.5^{\mathrm{d}}$ & 0.0 \\
\hline \multirow[t]{2}{*}{3} & 180 & 13.5 & 7.1 & 13.5 & 4.1 \\
\hline & & & & $8.0^{\mathrm{e}}$ & 0.0 \\
\hline 4 & 540 & 30.6 & 12.0 & 30.6 & 5.0 \\
\hline
\end{tabular}

8. ${ }^{\mathrm{e}}$ If counts $\leq 10$. possible using the full range of MOLA returns, from the minimum detectable to the strongest. Specifically, we seek to determine the duration of the pulse $W_{y}$ as a function of counts. The pulse energy measurement $A_{y}$, the area of the detected signal integrated while voltage exceeds the threshold $y$, is assumed accurate. The measured pulse width $W_{y}$ must satisfy

$$
W_{y} \leq \frac{A_{y}}{y} .
$$

[9] Figure 2 shows an array of ground return pulse width upper bounds vs. counts for channel 1 . Some points in the array exhibit shorter pulse widths than the calibration curve, since these measurements have low energy and are noisy. A tight lower bound on the pulse width is provided by the system impulse response characteristics. The laser pulse may be modeled as a Gaussian amplitude with respect to time, with a standard deviation $\sigma_{l}=3.5 \mathrm{~ns}$. Each 5-pole Bessel low-pass filter's impulse response at FWHM (full width at half maximum) is given in Table 1 . The response may be closely approximated by a Gaussian function with a standard deviation $\sigma_{f}=0.425$ FWHM, or $8.5 \mathrm{~ns}$ on channel 1. Combining the optical impulse response of surface terrain $\sigma_{t}$ with that of the laser and system, the variance of the pulse shape received at the discriminator is

$$
\sigma_{r}^{2}=\sigma_{t}^{2}+\sigma_{l}^{2}+\sigma_{f}^{2}
$$

and is approximately Gaussian. The output pulse shape as a function of time is:

$$
g(t)=\frac{A}{\sqrt{2 \pi} \sigma_{r}} \exp \left[-\frac{1}{2}\left(t / \sigma_{r}\right)^{2}\right],
$$

where $A$ is the area of the pulse in volt-nanoseconds. We assume a minimal pulse width, i.e., $\sigma_{t}=0$. Given $A_{y}$ and $\sigma_{r}$, the pair of equations [Abshire et al., 2000, Equations 12 and 14]

$$
W_{y}=2 \sigma_{r} \sqrt{-2 \ln \left(\frac{\sqrt{2 \pi} y \sigma_{r}}{A}\right)}
$$

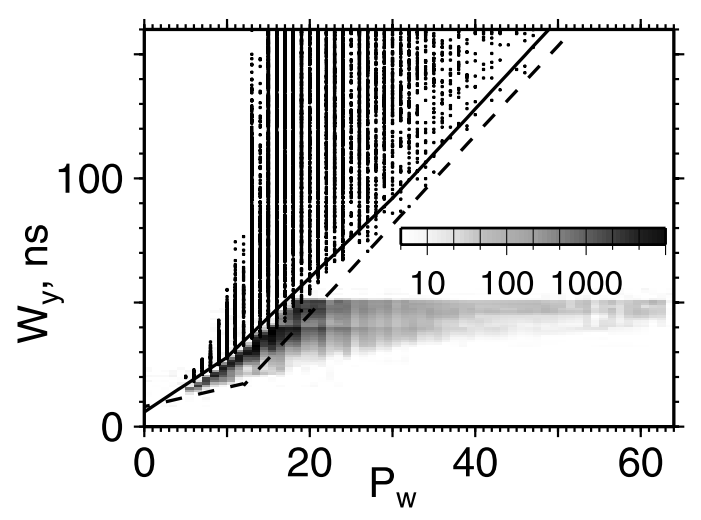

Figure 2. Preflight channel 1 pulse width calibration (dashes) and recalibration from this study (solid lines). Symbols plot maximum pulse width possible for a given threshold and energy, for shots over the course of a day. Grayscale region shows an array of pulse width lower bounds from system response obtained by solving Equations 5 and 6. Density ranges from 1 to 10,000 shots per day. 


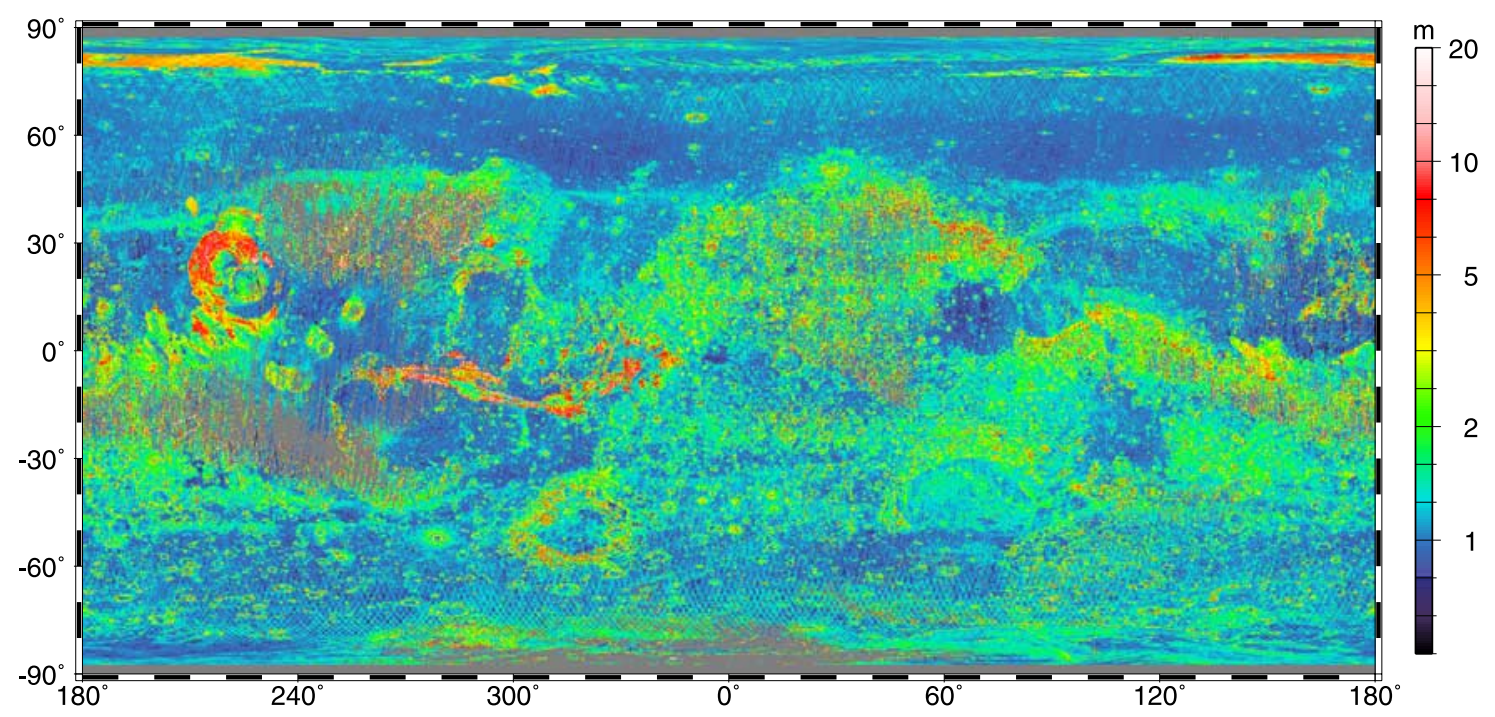

Figure 3. Terrain RMS roughness after correcting for slope, averaged over $1 / 4$ degree regions, in cylindrical projection. Logarithmic color scale shows rougher regions in warmer hues, while areas with no unsaturated data are shown in gray.

$$
A_{y}=A \operatorname{erf}\left(\frac{\mathrm{W}_{\mathrm{y}}}{2 \sqrt{2} \sigma_{r}}\right)
$$

may be solved numerically for $A$ and a predicted value of $W_{y}$. For a given measured energy, the measured pulse must be wider than given by Equation 5 since we have assumed no pulse spreading due to terrain. Figure 2 shows the occurrence of these bounds for ground pulses as a density plot. Our preferred calibration is consistent with both the system response and the threshold discriminator bound (Equation 2). The revised values of $W_{y}$ given in Table 1 as piecewise-linear functions of counts for each channel.

\section{Inversion for Terrain Response}

[10] If $W_{y}$ and $A_{y}$ are known, the RMS pulse width $\sigma_{r}$ may be obtained by Abshire et al. [2000, Equation 18]. Using the known system response on a given channel and Equation 3, we may solve for the terrain response $\sigma_{t}^{2}$, provided $\sigma_{r}^{2}$ is sufficiently large. The echo pulse width over flat Martian terrain is often narrower than the receiver impulse response. In cases where $\sigma_{r}$ is small Equation 3 has no real solution.

[11] To avoid such a singularity, we solve directly for $\sigma_{t}$ using the nonlinear total least-squares algorithm, wherein the parameters and observations are considered a random variable $\mathbf{x}$, with covariance $\mathbf{C}_{\mathbf{x}}$, related by an implicit theory $\mathbf{f}(\mathbf{x})=\mathbf{0}$. This theory is given by the amplitude and area equations for an ideal Gaussian pulse waveform:

$$
\begin{gathered}
f_{1}=\frac{A}{\sqrt{2 \pi} \sigma_{r}} \exp \left[-\left(\frac{W_{y}}{2 \sqrt{2} \sigma_{r}}\right)^{2}\right]-y=0 \\
f_{2}=A \operatorname{erf}\left(\frac{\mathrm{W}_{\mathrm{y}}}{2 \sqrt{2} \sigma_{\mathrm{r}}}\right)-\mathrm{A}_{\mathrm{y}}=0
\end{gathered}
$$

with an associated (diagonal) covariance $\mathbf{C}_{\mathrm{f}}$ that accounts for noise in the waveform equal to $10 \%$ of threshold $y$, and deviation of $1 \mathrm{~V}$ ns, about $1 \%$ of the typical energy received, from the area under an ideal Gaussian shape.

[12] Consistent with observations over Martian terrain, and to ensure positive values, we assume that $\sigma_{t}$ is lognormally distributed with a standard deviation equivalent to 20fold uncertainty, or 3 units in log space. The a priori value is proportional to the system response of each channel, as the shortest pulses trigger earlier on channels with shorter response. Pulse energy is also approximately lognormally distributed, with an expected value close to $A_{y}$ and 10 -fold uncertainty. The combined parameter and data vector $\mathbf{x}$ is given by $x_{1}=\log (A), x_{2}=\log \left(\sigma_{t}\right), x_{3}=W_{y}$, and $x_{4}=A_{y}$. The standard deviations of the observations are set to one LSB of the QT100 counters. A damped, nonlinear, quasi-Newton method converges to the maximum-likelihood estimate $\hat{\mathbf{x}}$. The solution at the $(k+1)^{\text {th }}$ iteration is [Tarantola, 1987, 1.143]:

$$
\mathbf{x}_{k+1}=\mathbf{x}_{k}-\left[\mathbf{F}^{\mathbf{T}} \mathbf{C}_{\mathrm{f}}^{-1} \mathbf{F}+\mathbf{C}_{\mathrm{x}}^{-1}\right]^{-1}\left[\mathbf{F}^{\mathbf{T}} \mathbf{C}_{\mathrm{f}}^{-1} \mathbf{f}+\mathbf{C}_{\mathrm{x}}^{-1}\left(\mathbf{x}_{k}-\mathbf{x}_{0}\right)\right],
$$

where $\mathbf{F}=\left[\partial f_{i} / \partial x_{j}\right]$ is the matrix of partial derivatives evaluated at the $k^{\text {th }}$ iteration, $\mathbf{f}=\left[f_{i}\left(\mathbf{x}_{k}\right)\right]$, and the superscript $\mathbf{T}$ denotes the matrix transpose. At $\hat{\mathbf{x}}$, a formal posterior estimate of covariance approximates the true (non-linear) covariance:

$$
\mathbf{C}_{\hat{\mathrm{x}}} \simeq\left[\mathbf{F}^{\mathbf{T}} \mathbf{C}_{\mathrm{f}}^{-1} \mathbf{F}+\mathbf{C}_{\mathrm{x}}^{-1}\right]^{-1}
$$

Assuming maximal unsaturated pulse area counts, this equation predicts that channel 1 pulses can resolve terrain response as low as $1 \mathrm{~m}$ (6.67 ns) RMS, and can provide $20 \%$ uncertainty in response over a range of 3-30 m RMS. Our solution closely matches that of Abshire et al. [2000], but without singularities at the lowest and highest counts. 


\section{Interpretation of Surface Roughness}

[13] A terrain response function and its variance has been given by Gardner [1992]. Where the laser beam pattern is a bivariate Gaussian with a small RMS divergence angle $\gamma$, neglecting the effects of shot noise, speckle, wavefront curvature, and forward scattering through clouds, we write as a special case

$$
\sigma_{t}^{2}=\sigma_{m}^{2}+\frac{4 R_{m}^{2}}{c^{2}}\left[\tan ^{2}(\gamma) \tan ^{2}(\theta)\right]
$$

where $\sigma_{m}^{2}$ is due to height variability of Mars within the footprint after a regional slope is removed, $R_{m}$ is the laser one-way range, and $\tan \theta$ is the surface slope with respect to the incident laser beam. As nearly all MOLA observations are at nadir, we equate the effect of slope over long baselines to that of oblique incidence.

[14] The MOLA laser beam pattern departed from the Gaussian ideal in Equation 11. The nominal divergence $\gamma=$ $93 \mu \mathrm{rad}$ [Abshire et al., 2000] predicts larger pulse widths over sloping terrain than were observed. We used inflight data and the Gardner [1992] model to estimate $\gamma$, finding that the divergence was $\sim 0.5$ times nominal, owing to hot spots in the laser beam. The effective laser footprint was about $75 \mathrm{~m}(2 \sigma)$ at an average $R_{m}=400 \mathrm{~km}$.

[15] Using our preferred value of $\gamma$, we remove the predictable component of terrain response due to regional slope, sampled from a $1 / 64^{\circ}$-per-pixel grid, to obtain an RMS roughness, scaled to meters. Contributions to pulse spreading from short-baseline slopes not resolved by regional models might be removed using footprint-to-footprint slope estimates, but these estimates neglect acrosstrack gradients and underestimate $\tan (\theta)$.

[16] Unsaturated pulse widths corrected for $\mathrm{km}$-scale slope (Figure 3) show the exceptional smoothness of the northern lowlands; Utopia, Amazonis, and Elysium Planitia have a modal roughness of about $1 \mathrm{~m}$ RMS, broken by rougher ejecta of large craters and bordered by the dichotomy boundary. While the lowlands are also smooth at baselines of $0.6 \mathrm{~km}$ and longer [Aharonson et al., 1998], some topographically benign regions are much rougher at footprint scale, such as the $\sim 5 \mathrm{~m}$ RMS teardrop-shaped region at $208-212^{\circ} \mathrm{E}, 37-43^{\circ} \mathrm{N}$. Apart from understandably rugged terrains such as the Olympus Mons aureole deposits, the walls of Valles Marineris, and large crater rims, the most prominent regions of high roughness appear to be the dunes in the Vastitas Borealis. Polar deposits are generally smooth, but noticeably rougher in the south in the vicinity of Chasma Australe, where sublimation pits and aeolian scouring are seen in images and point-to-point profiles.

[17] Slope-corrected pulse widths provide a unique measurement of roughness at a length scale of 10's of meters that is of interest for landing studies. The Terra Meridiani region soon to be visited by one of the 2003 Mars Exploration Rovers, as well as Syrtis Major, are among the smoothest parts of the highlands at footprint scale. The majority of pulse widths over the four rover candidate landing sites are below our 1-m detection limit. Nearly $95 \%$ of pulses at the Meridiani site are below $1 \mathrm{~m}$, but three other sites, in Isidis Basin, Gusev Crater, and Elysium Planitia, have about a $5 \%$ chance of encountering $>3 \mathrm{~m}$ RMS roughness within a given footprint.

[18] A latitudinal trend toward lower roughness both north and south appears in Figure 3, as noted by studies of km-scale values [Kreslavsky and Head, 2000] and correlation length [Aharonson et al., 2001]. The mid-latitude transitional band of $\sim 2$-m roughness stretching north of Tharsis at $35-50^{\circ} \mathrm{N}$ and skirting Argyre and Hellas at $35-50^{\circ} \mathrm{S}$ stands out clearly at footprint scale. The enhanced fine-scale roughness of this terrain is attributed by Mustard et al. [2001] to devolatilization of ground ice. If indeed past variations in orbital parameters are responsible for the geomorphic signature, then the latitudinal boundaries delineated by MOLA pulse-width may constrain the nature of the presumed event.

[19] Acknowledgments. The MOLA investigation is supported by the NASA Mars Exploration Program. Constructive and thorough reviews by F.S. Anderson and N. Bridges improved the manuscript.

\section{References}

Abshire, J. B., X. Sun, and R. S. Afzal, Mars Orbiter Laser Altimeter: Receiver model and performance analysis, Appl. Opt., 39, 2440-2460, 2000 .

Aharonson, O., M. T. Zuber, G. A. Neumann, and J. W. Head III, Mars: Northern Hemisphere slopes and slope distributions, Geophys. Res. Lett., 25, 4413-4416, 1998.

Aharonson, O., M. T. Zuber, and D. H. Rothman, Statistics of Mars' topography from the Mars Orbiter Laser Altimeter: Slopes, correlations, and physical models, J. Geophys. Res., 106, 23,723-23,735, 2001.

Gardner, C. S., Ranging performance of satellite laser altimeters, IEEE Trans. Geosci. Remote Sens., 30, 1061-1072, 1992.

Garvin, J. B., J. L. Bufton, J. B. Blair, D. Harding, S. B. Luthcke, J. J. Frawley, and D. D. Rowlands, Observations of the Earth's topography from the Shuttle Laser Altimeter (SLA): Laser pulse echo recovery measurements of terrestrial surfaces, Phys. Chem. Earth, 23, 1053-1068, 1998.

Garvin, J. B., J. J. Frawley, and J. B. Abshire, Vertical roughness of Mars from the Mars Orbiter Laser Altimeter, Geophys. Res. Lett., 26, 381-384, 1999.

Kreslavsky, M. A., and J. W. Head III, Kilometer-scale roughness of Mars: Results from MOLA data analysis, J. Geophys. Res., 105, 26,69526,711, 2000.

Mustard, J. F., C. D. Cooper, and M. K. Rifkin, Evidence for recent climate change on Mars from the identification of youthful near-surface ground ice, Nature, 412, 411-414, 2001.

Smith, D. E., et al., Mars Orbiter Laser Altimeter: Experiment summary after the first year of global mapping of Mars, J. Geophys. Res., 106, 23,689-23,722, 2001

Tarantola, A., Inverse Problem Theory, Elsevier Sci., New York, 1987.

Zuber, M. T., D. E. Smith, S. C. Solomon, D. O. Muhleman, J. W. Head, J. B. Garvin, J. B. Abshire, and J. L. Bufton, The Mars Observer Laser Altimeter investigation, J. Geophys. Res., 97, 7781-7797, 1992.

J. B. Abshire, G. A. Neumann, and X. Sun, Laboratory for Terrestrial Physics, NASA/Goddard Space Flight Center, Building 33, Greenbelt, MD 20771, USA. (neumann@tharsis.gsfc.nasa.gov)

O. Aharonson, Department of Geological and Planetary Sciences, California Institute of Technology, 170-25, Pasadena, CA 91125-2500, USA.

J. B. Garvin, NASA Headquarters, Washington, DC 20546, USA.

M. T. Zuber, Department of Earth, Atmospheric and Planetary Sciences, Massachusetts Institute of Technology, 54-518, Cambridge, MA 021394307, USA. 